\title{
Early-type Galaxy Formation History from GALEX-SAURON
}

\author{
Hyunjin Jeong ${ }^{1}$, Sukyoung K. Yi ${ }^{1}$, Martin Bureau ${ }^{2}$, \\ Davor Kranović ${ }^{2}$ and Roger L. Davies ${ }^{2}$ \\ ${ }^{1}$ Department of Astronomy, Yonsei University, Seoul 120-749, Korea \\ email:hyunjin@galaxy.yonsei.ac.kr \\ ${ }^{2}$ Sub-Department of Astrophysics, University of Oxford, Oxford OX1 3RH, UK
}

\section{Introduction}

One of long-standing debates in modern astrophysics is the formation mechanism of early-type galaxies. The classical model, proposed by Eggel et al. (1962), explains that early-type stellar populations form in an initial highly efficient burst and evolve without further star formation until present day. The high $\mathrm{Mg}$ and alpha abundances found in bright elliptical galaxies support such scenarios. Early-type galaxies, therefore, are traditionally believed that they are dynamically simple stellar systems with homogeneous stellar populations (e.g. Gott 1977). The popular Lambda Cold Dark Matter (LCDM) paradigm (e.g. Toomre and Toomre 1972), however, strongly suggested a hierarchical merger picture for massive elliptical galaxies. In this model, early-type galaxies form as a result of major mergers and are thought to have continued star formation. Evidence is growing that a substantial fraction of early-type galaxies has secondary star formation. Furthermore, SAURON survey has revealed a rich diversity in the kinematics, discovering numerous central disks and kinematically decoupled cores (e.g. Emsellem et al. 2004; Sarzi et al. 2006). Early-type galaxies are thus likely to have had complex and varied formation histories.

Unlike optical bands, ultraviolet (UV) light is very sensitive to stellar populations younger than a few hundred megayears. Therefore, UV is a good tracer to study young stars in old galaxies. With Galaxy Evolution Explorer (GALEX) satellite (see Martin et al. 2005; Morrissey et al. 2005) it is possible to search for hint of star formation by combining with existing kinematic and stellar population data from SAURON.

\section{Observations and Results}

We have been observing early-type galaxy sample from the SAURON project with the imaging mode of GALEX and the MDM Observatory 1.3-m McGraw-Hill Telescope. Some are centrally concentrated while others are extended. We even have seen a case where a classical elliptical galaxy NGC2974 exhibits a magnificent UV ring $6.2 \mathrm{kpc}$ away from its centre coinciding with the Outer Lindblad Resonance (OLR) generated by the bar. This large-scale bar is confirmed by small peak in UV surface brightness profiles and resonance diagram. Fig. 1 shows the GALEX FUV, NUV, MDM F555W and MDM F555W unsharp-masked images of NGC2974. The optical image has the smooth appearance, but the UV images show a complete ring in the outer parts.

Blue FUV - NUV and UV - optical colours are also observed in many early-type galaxies meaning the presence of young stellar populations and recent star formation (RSF). This is supported by a simple stellar population model which assumes two bursts 

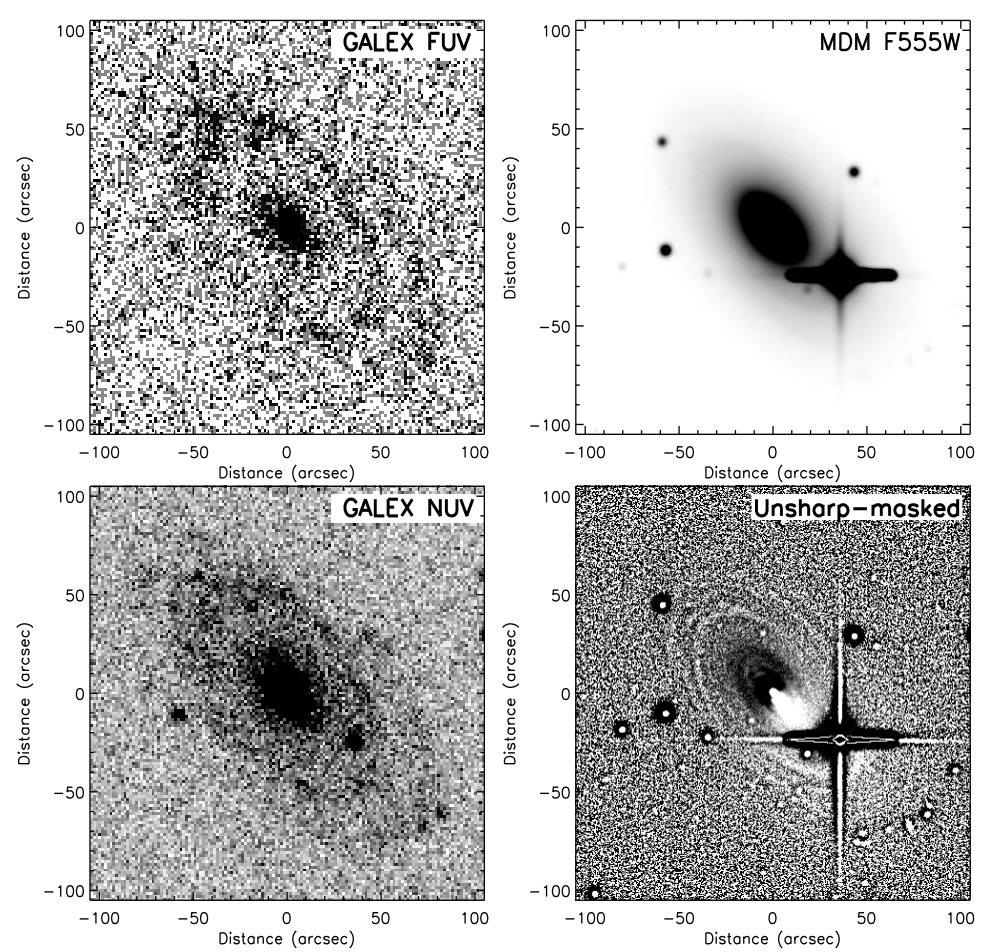

Figure 1. GALEX UV and MDM optical images of the central $100 \operatorname{arcsec} \times 100 \operatorname{arcsec}$ of NGC 2974. Note the bright foreground star to the South-West.

of star formation, allowing us to constrain the age, mass fraction and surface mass density of the young component pixel by pixel.

\section{Conclusions}

Early-type galaxies are not dynamically simple systems with homogeneous stellar populations anymore. Our recent sample NGC2974, known as an E4 elliptical in the optical band, reveals a grand star-forming ring in the UV images. Blue UV - optical colours are also observed in many early-type galaxies suggesting the presence of young stellar populations and RSF. This suggests that star formation may thus not be all that unusual in early-type galaxies, or perhaps elliptical galaxies in the classical sense are simply far rarer than usually assumed.

\section{Acknowledgements}

We would like to acknowledge that this work was supported by grant from KOSEF.

\section{References}

Eggen, O. J., Lynden-Bell D., Sandage A. R. 1962, ApJ 136, 748

Emsellem E. et al. 2004, MNRAS 352, 721

Gott J. R. 1977, ARA\& A 15, 235

Martin D. C. et al. 2005, ApJ(Letters) 619, L1

Morrissey D. C. et al. 2005, $A p J$ (Letters) 619, L7

Sarzi M. et al. , MNRAS 366, 1151

Toomre A. and Toomre J. 1972, ApJ 178, 623 